\title{
Il suicidio di Saffo trattato da Grillparzer, Leopardi e Pavese
}

\author{
Grazia Sotis
}

La critica moderna ha rivolto molta attenzione alla figura di Saffo. Attraverso le sue poesie la poetessa di Lesbo cantò se stessa, rompendo cosí il silenzio dell'io femminile nell'antichità classica. Saffo visse in un periodo di transizione. Nel settimo secolo il dominio della vecchia aristocrazia venne scosso dalla nascita di una nuova classe sociale, quella mercantile e commerciale. In questo stato di cose la poctessa, secondo quanto riferisce Page duBois (95-106), liberò se stessa e quindi le altre donne dal rigido ruolo dato loro da una lunga tradizione che vedeva solo l'uomo come eroe.

Eva Stchle in "Sappho's Private World," presentando una donna con un io prettamente femminile, visto non piú come un oggetto dell'eroticità maschile bensí come un soggetto erotico, arricchisce lo studio analitico delle poesie di Saffo fatto da Richard Jenkyns e Anne Pippin Brunett, i quali hanno messo in evidenza una poetessa che aveva una sensibilità moderna e per questa ragione diversa dai poeti del suo periodo. Secondo Jenkyns, Saffo

depicts the imprecise with great precision. She looks at her sorroundings through half-shut eyes .... It is precisely because Sappho is, within her limits, so remarkable sensitive to natural impressions that we are liable, unless we are careful, to attribute to her a modern sensibility. (40)

Da un punto di vista poetico, secondo F. Della Corte, Saffo "ha rinunciato al senso positivo dell'esistenza, e, con l'indagine introspettiva che penetra nel profondo della sua psiche, anziché comporre la sua figura in un atteggiamento statuario, ha voluto scompigliare i suoi gesti, sfuggire al rigore di un comportamento statico, rovesciare il passato epico in un presente esteticamente lirico" (23).

Tre poeti moderni hanno contribuito in modo particolare a proiettare la figura di Saffo nel nostro mondo, gettando una nuova luce su una poetessa alle prese con un io prettamente femminile ancor 
prima di quanto stabilito dalla critica di oggi: Grillparzer, Leopardi e Pavese. I tre scrittori nel cantare Saffo hanno avvertito un'affinità elettiva da ricercare non tanto nell'amore infelice quanto nell'artista alla ricerca di sé. Per tutti e tre Saffo rappresenta il prototipo dello artista moderno che viene a conoscenza del proprio essere non solo di poeta ma anche di donna.

Grillparzer nella tragedia Sappho (1818) tratta il motivo da cui è scaturito il tormento della donna: l'amore non corrisposto; ed evidenzia la disperazione di un animo che si trova a combattere contro forze oscure: irrazionalità, gelosia, rabbia. In lei prevale e s'impone un alter ego, con cui stenta ad identificarsi e che non sa accettare come espressione di se stessa. Leopardi nell" "Ultimo canto di Saffo" (1822) va invece aldilà del tema dell'amore, in una ricerca dell'infelicità esistenziale della donna, alla quale unisce il suo canto e la sua voce di poeta. Pavese in "Schiuma d'onda" (1947) è alle prese con la delusione della donna che nella morte aveva cercato una soluzione alla sua esistenza terrena.

I tre lavori modulano il tema del suicidio in fasi diverse. Mentre per Grillparzer diventa un atto nobile che riscatta Saffo dall'essere diventata una donna, ridimensionando il mito creatosi intorno all'antica poetessa per conferirle un aspetto piú femminile, per Leopardi è la conseguenza della disperazione e della negatività nei riguardi della vita. D'altro canto, Pavese impernia il dialogo sull'inutilità e sulla noia della morte, sul vuoto esistenziale vissuto come una realtà vera, eterna, alluccinante.

A differenza della Saffo di Pavese e di Leopardi, quella di Grillparzer è violenta nelle sue manifestazioni. Questo ultimo affronta per primo uno studio psicologico su un personaggio rendendolo vivo: lo scrittore austriaco non ha voluto creare una "cosa dell'immaginazione" ("eine Sache der Imagination"), ma una donna vera, dibattuta. Anche se nella scena iniziale dell'opera è presentata nella pomposità e gloria di poetessa, è tuttavia una semplice donna innamorata, timorosa di perdere l'amicizia e l'amore di Faone, e avverte il tumulto creatole da questo affetto per l'uomo. Chiaramente lui non corrisponde l'amore della donna ma della poetessa, anzi ne era rimasto affascinato prima ancora di averla vista, attraverso la lettura delle poesie. Ironicamente la Saffo donna non piace ai suoi servitori, forse neppure a Melitta, la giovane schiava cara alla poctessa e alla 
quale ricorda il suo stesso passato di fanciulla:

ancora timida, con le gote tonde di una bambina, desiderando vagamente nel mio petto tormentato, pesante, camminavo il mondo libero con passi sciolti l'illusione ancora, non conoscenze con le sue torture, era tutta la musica delle mic corde dorate; quando l'amore era ancora un terreno fantastico, vergine, una magica terra stranicra. (Atto I, scena 5)

Il diaframma che separa la fanciullezza dalla maturità viene spezzato bruscamente al momento della conoscenza che fa cadere l'illusione, il velo di Maía, del mondo per aprirci una realtà tormentata e un vuoto. La donna di Grillparzer si rende subito conto dell'impossibilità di un amore simile, sí da descriverlo come qualcosa che si intuisce con un senso di sbigottimento ("il mio sguardo può misurare il vuoto, ma non il mio piede"), come colui che s'avventura con una barca leggera per mondi sconosciuti circondato da "una grigia estensione di spazio senza limiti. Vede la sponda assolata, ma spostata lontana."

Nella seconda scena, la donna s'accorge dell'innamoramento dell' uomo per la giovane schiava, e inizia cosí la sua sofferenza, "Come le stava vicino! Come era caldo il suo abbraccio! . . . . Lei sulle sue labbra - via! Non voglio pensarci!" Ad aumentare il suo tormento sopravviene il dubbio, se fosse stato giusto, per lei grande poetessa, innamorarsi di Faone. La gelosia è ingigantita se vista in rapporto all'innocenza e alla calma di Melitta che come una novella Nausica

s'è avviata verso il ruscello che scorreva

limpido e fresco attraverso il boschetto di mirto. (Atto III, scena 3)

Saffo affronta Melitta in un attimo di rabbia. La reazione di Faone la accentua maggiormente quando lui dichiara apertamente di sentirsi liberato dall'incantesimo ora che la donna gli si è manifestata nella sua nudità. Colpita, Saffo piange senza destare alcuna compassione sull'uomo che continua a vederla come una Circe.

In un primo tempo la donna si sente tradita dalle persone che riteneva degne del suo affetto e vorrebbe che il mondo sprofondasse con lei; poi è invasa dall'ira che le preclude di essere ragionevole, al punto di voler uccidere Melitta, degradandosi cosí sempre di piú davanti a Faone. I due giovani cercano di fuggire ma vengono catturati. Le parole terribili dell'uomo riportano la donna ai suoi sensi 
Solo una donna posso pensare tanto crudele, una donna debole, profondamente e vilmente arrabbiata. Sei stata tu a colpirla; quanto ti conosco ora! Via da me, via! (Atto IV, scena 3)

e Saffo cerca scampo, ma non dall'uomo, come lui crede: "Fuggi da me? Prima rispondi alle mie accuse! Ah, tremi! E ora che tu tremi ed abbi paura!" ma da se stessa. Le offese di Faone si susseguono con un ritmo incalzante

I pregi dorati della poesia sono andati perduti.

Non profanerai piú il nome dell'arte:

Ma tu hai usato la poesia come veleno per causare una morte crudele al tuo nemico:

Quanto diversa è la Saffo che avevo creduto

Chi ti ha trasformato con la sua bacchetta magica? (Atto IV, scena 3)

La trasformazione di Saffo è avvertita attraverso le terribili parole dell'uomo che testimoniano il precipizio in cui la donna era caduta. L'atteggiamento di Faone è tuttavia molto ambiguo: la sua sembrerebbe la presa di posizione di colui che vuole far sentire la propria forza e punire con essa la debolezza umana e femminile di Saffo. Confrontandosi con lui, lei intravede come in uno specchio il proprio animo, l'uomo diventa l'ostacolo, o il punto fermo che porta la donna a una riflessione non tanto del proprio agire, quanto del proprio carattere. Inizialmente Saffo lo aveva avvertito di quello che era capace, cioc̀ di azioni inaspettate; forse in fondo era consapevole della potenzialità del suo io, soltanto non aveva mai trovato il punto di misura e l'ostacolo che la fermasse a riflettere sulla sua personalità: essere una donna prima ancora di una grande poetessa. Con le sue accuse, Faone la spoglia del vestito poctico per mostrarcela in tutta la sua femminilità. La Saffo di Grillparzer non è poi cosí diversa da quella greca che nelle sue poesie andava cantando i suoi desideri, debolezze . . . l'amore, e aveva messo in mostra il suo animo rendendosi diversa dagli altri poeti dell'antichità classica. La Saffo di Grillparzer è in parte un moderno Orfeo che, andato alla ricerca di Euridice, ha invece trovato se stesso. Saffo dirà a Faone: "Cercavo te, e ho trovato me stessa." Ed è Saffo a non perdonare se stessa dall'essere caduta in preda all'elemento irrazionale della sua personalità; chiede perdono a tutti quelli che la hanno vista nella sua 
debolezza umana, eccetto a se stessi. Lei non si perdona, si punisce per tornare pura "alle acque del mare," alla poesia e agli dei, "a quelli che sono come lei," dirà Rhames a conclusione della tragedia. Cosí facendo Saffo ha ritrovato la compostezza che si addice a un animo nobile.

Secondo alcuni critici, la Saffo di Grillparzer non è riuscita a colmare la discrepanza fra arte e vita. L'intento artistico dello scrittore era stato quello di creare una donna in carne e ossa, una donna che, una volta scoperta se stessa e la realtà dei suoi sentimenti, aveva cercato di fuggire da sé. L'arte non è certamente riuscita a soffocare la personalità prettamente femminile di Saffo; tutt'altro, lei vi ha trovato la sua natura di donna.

Nella letteratura tedesca della Germania Saffo rappresentava un elogio alla femminilità classica e una difesa delle donne tedesche contro gli assalti del decadentismo dei francesi. Ma a differenza della letteratura tedesca della Germania, Grillparzer con Sappho ha sconvolto i valori di equilibrio, bellezza, serenità classica professati dal Winckelmann, creando cosí una donna vera con tutta la problematica congenita in una situazione che l'ha spinta al suicidio. La maggior parte della critica ha cercato in Sappho una linea di scrittura, da parte di Grillparzer, fine a se stessa. Allontanandosi dalla letteratura della Germania, l'intento dello scrittore è stato di fare della donna un'eroina, non perché fosse la grande Saffo, ma perché è riuscito a scoprire prima di tutto un io pieno di contraddizioni: rivelazione che sconvolge la Saffo austriaca a un punto tale da cercare la morte per fuggire da sé e non da Faone; la rabbia, l'ira, l'irrazionalità di cui era capace, sono forme congenite della sua personalità.

Quanto diversa dalla Saffo austriaca è quella cantata da Leopardi! Il pocta italiano la vede con gli occhi di un romantico e come un tramite dal suo io poetico. Saffo s'impone al poeta, come sarà per Pavese, come artista infelice. La natura, come la dea del mare in Pavese in cui Saffo si è trasformata, è indifferente, e il ciclo monotono delle stagioni conduce il poeta al "nulla eterno." Lo scoglio del mare, sia in Pavese, sia in Grillparzer, come la siepe dell' "Infinito," è, per dirla con Luti, a proposito della siepe leopardiana, l' "ostacolo su cui si misura la proiezione verso la liberazione e la conoscenza dell'io poetico" (in Lonardi 16). L' "Ultimo canto di Saffo" ì la voce dello stesso Leopardi che, nella figura della donna, riesce a 
trovare e a misurare il suo coraggio verso un salto nel vuoto.

Il canto si svolge con un'apparente tranquillità, in una "placida notte." Ma già dall'inizio c'è un accenno alla irrequietezza del poeta, "mentre ignote mi fur l'erinni e il fato." Inoltre, la presenza della rupe porta direttamente a un legame del poeta con Saffo: secondo la leggenda, la poetessa di Lesbo si buttò nel mare da una rupe. (Ne "La rupe" dai Dialoghi con Leucò, Pavese fa dire a Prometeo che tutti gli uomini hanno una rupe.)

Anche Saffo aveva cantato la bellezza e l'amore, purtroppo la placida notte l'ha sempre trovata un'anima in pena. Nella prima parte, per libera associazione, c'è già in embrione il riferimento al "flutto" marino, in virtú del movimento ondeggiante di un campo di messi. Dalla placida notte si passa, in termini psicologici attraverso l'uso di immagini concrete, all'irrequietezza di due spiriti affini. "Noi" indica la comunione avvenuta e getta luce sulle immagini seguenti: "la dubbia sponda" e "l'ira dell'onda." Il ricordo costituisce un ruolo importante nella poesia di Leopardi. Il passato diventa vago, incerto, come pure incerta o mal sicura è l'onda. La descrizione iniziale da punti fermi ben determinati (luna, selva, rupe) prende il balzo per un sentimento indeterminato: l'unione con la poetessa è quindi piú facile, resa possibile proprio da questo salto verso una forma meno definita (aere, profondi, vasta), quello che resta è un'emozione di sbigottimento.

La realizzazione di Saffo, di non far parte della bellezza del mondo, origina un senso di negatività e di comunione con l'io leopardiano evidenziato dall'uso di "non": "non ride" "non il canto" "non dei faggi" per cui, nell'ultima parte, le immagini sono avvertite fuggevoli: non è Leopardi/Saffo a fuggire la vita, ma al contrario, loro la vogliono, purtroppo è la vita che fugge loro. "L'aprico margo" non porta alla pienezza della vita ma alla sua negazione. Una donna artista rappresenta l'incapacità di ribellione da parte di Leopardi. In "Bruto Minore," un'altra poesia dove Leopardi tratta del tema del suicidio, Bruto, suicida anche lui, non è uno spirito affine a quello del poeta in quanto capace di una "titanica ribellione," Saffo, invece, con la sua condizione sociale e biologica, esprime meglio l'accettazione e sottomissione all'ordine cosmico, lei esprime meglio l'aspetto piú intimo, piú nascosto del poeta recanese.

L'identificazione di Leopardi con Saffo è accentuata nella terza 
parte della poesia. Ora è la voce femminile a prendere il sopravvento e si chiede del perché della sua infelicità. Misterioso è il destino dei due poeti: "arcano ì tutto" e, secondo De Robertis, la stessa parola genera paura in quanto ingigantita dall'uso insistente che ne fa Leopardi. La poesia rende i due poeti coscienti della loro infelice situazione: l'artista va alla ricerca della sua ragion d'essere e scopre invece il vuoto in sé e intorno a sé. "Morremo": è la coscienza del suicidio avvenuto e ancora da venire. Saffo è la morte "mitica" di Leopardi avvenuta al momento dell'epifania, del nulla spaventoso. La bellezza non è una realtà: aldilà del velo di Maia c'è il nulla, il vuoto, il silenzio. Se per Schopenhauer l'artista nel contemplare l'arte perde la sua individualità, non è cosí per Leopardi, il quale, attraverso l'arte, afferma la sua individualità, l'io poetico in tutta la sua forza espressiva di negatività. Saffo ha dato a Leopardi lo spunto per parlare di se stesso, della sua infelicità di artista, come l'antica poetessa aveva già fatto ai tempi suoi. Lei, attraverso la sua infelicita di donna, aveva raggiunto quella dell'artista, la cui arte non era conforme alla moda del periodo. Saffo ha oscultato il suo io femminile pieno di contraddizioni, di emozioni contrastanti che l'hanno resa piú umana, piú vera, piú viva. Come Leopardi, anche Saffo ha parlato delle sue emozioni in momenti di "incertezza."

Jerkyns ha messo in evidenza il legame che c'è fra i poeti romantici inglesi, soprattutto Wordsworth, e la poetessa greca: cioc̀ la loro capacità di evocare una sensazione. Nell" "Ultimo canto di Saffo" Leopardi evoca sensazioni attraverso una poesia ricca di immagini ben precise che poi vengono sfocate e controbilanciate da sentimenti persi nel tempo e nello spazio. Quindi, un'affinità fra Lcopardi c Saffo è da ricercare in una tecnica poetica.

La poetessa di Lesbo rappresenta la scoperta dell'io poctico. Grillparzer rende la sua Saffo consapevole dei propri limiti, debolezze c paure: il suicidio non è il risultato dell'inconciliabilità della sua arte con la vita, quanto una presa di coscienza sulla natura della sua personalità. Inoltre, lei rappresenta un aspetto dell'io esistenziale espresso dall'inutilità della corona d'alloro in Grillparzer, che non ha certamente aiutato la donna a conquistare Faone; dal vuoto in Leopardi ("e l'atra notte, e la silente riva"); dal movimento ondoso in Pavese, di cui parleremo in seguito.

Per un discorso sulla Saffo di Pavese conviene tener presente il ro- 
manzo Tra donne sole (1949) uscito due anni dopo la pubblicazione dei Dialoghi. Saffo e Britomarti di "Schiuma d'onda" sono aspetti della personalità di Pavese, e lo stesso si dica di Clelia, Momina e Rosetta di Tra donne sole. Le donne del dialogo hanno piú o meno le stesse caratteristiche di quelle del romanzo.

Saffo è descritta in termini di desiderio, inquietudine, tumulto. Il suicidio non ha risolto la sua infelicità, piuttosto è stato una delusione perché si rende conto che a nulla si sfugge.

In una sua poesia, Saffo, a differenza di Omero e di Alceo, aveva visto Elena non come una donna oggetto, o come un premio che si dava all'eroe, ma come una donna che agisce per conto proprio, che va alla ricerca della cosa che ama (Page duBois 95-106). Ed è questo aspetto di Elena cosí celebrato da Saffo ad interessare Pavese tale da diventare, per la Saffo pavesiana, un modello di comportamento in quanto non era fuggita da niente e da nessuno, è sempre stata uguale a se stessa, ma soprattutto bastava a se stessa: tutto ciò che la Saffo di "Schiuma d'onda" avrebbe voluto essere.

Britomarti è scappata dal bosco per fuggire un uomo e per rimanere se stessa (anche se da ninfa del bosco è diventata ninfa del mare). Ma Saffo, che si è chiesta il perché della vita, del suo essere, trova la risposta nel movimento sempre uguale e incessante delle onde e vede l'esistenza come qualcosa di monotono, inutile e terribilmente triste. Le onde sono l'essenza della donna e della dea del mare: esse rappresentano una vita eterna, uguale, monotona, ciclica, senza nessuno scopo o fine.

Un evento ciclico è ciò che accoglie Clelia a Torino, il Carnevale che per lei è triste perché le ricorda la morte del padre avvenuta durante un altro Carnevale. Nell'albergo dove alloggia Clelia una donna, Rosetta, ha cercato la morte. Il ritorno di Clelia a Torino è un tentativo di ritrovare se stessa ed è il desiderio della donna di ritrovarsi uguale. Purtroppo ella trova il vuoto intorno a sé epitomizzato nel negozio che era "sull'orlo del vuoto" (229), un po' come la siepe o la rupe leopardiana. Ma Clelia si difende e sopravvive volendo "bene a se stessa" (231).

Il primo tentativo di suicidio di Rosetta sembrerebbe causato da un rapporto di omoeroticità fra lei e Momina. Queste donne pavesiane sono libere, o liberate, solo apparentemente, in quanto rimangono poi prigioniere di se stesse. Se Clelia è libera volendo bene a se 
stessa, "trovando la sua regola di vita come scapola e prendendo gli uomini come noi si prende le donne" (Pavese, Lettere, 409), e Momina con il suo comportamento eccentrico, Rosetta è l'unica che cerca la libertà nella morte. Tuttavia quando fallisce il suo primo tentativo, è criticata duramente da Momina, che è la voce di Pavese critico severo, ma come quest'ultima lui è impotente e incapace di un atto simile. Rosetta dice che, dopo aver cercato la morte, tornare alla vita è piú difficile, vuol dire "stare peggio di prima. È questo che spaventa" (257). Anche Saffo cercando la morte non ha risolto il problema della sua esistenza, anche lei sta peggio di prima. Che cos'è la morte se non un vuoto che ti rende il risveglio orribile: Rosetta l'ha cercata per forse rompere la monotonia, il modo ondoso, il susseguirsi uguale $\mathrm{e}$ insopportabile delle sue giornate. Ma anche Momina vorrebbe morire, è vigliacca e procrastina aspettando la bella stagione per farlo. Rosetta, invece, vuole agire, non ha "pazienza di aspettare."

Saffo, per Pavese, è artista, ma soprattutto donna coraggiosa. Lei diventa un modello di coraggio che non aveva trovato in un uomo. O forse sí, in Walt Whitman, però quest'ultimo non aveva fuggito la vita. Whitman per Pavese rappresentava la pienezza dell'essere e della vita, tutto ciò che lui avrebbe voluto essere, come lui stesso afferma nel suo diario. Invece, Saffo di Lesbo è piú consona al carattere di Pavese: il tentativo di accettare se stessi e non cercare di essere diversi.

Nella Saffo di tutti e tre gli scrittori c'è un senso di smarrimento. In Grillparzer è trasmesso attraverso una descrizione del mare grigio, illimitato opposto alla descrizione amena del prato, dei campi dell'isola di Lesbo. Come in Leopardi cosí in Grillparzer lo smarrimento prende la forma di una poesia indefinita: campo contro mare in Grillparzer; rupe-siepe contro spazio (psicologico) in Leopardi; roccia contro onde in Pavese. La Saffo pavesiana accenna alla dea del mare, come quest'ultimo anche la dea è inafferrabile, cosí come lo è la vita.

Secondo Pavese, Saffo è astorica in quanto per lei, come per tutte le donne, non esiste la storia. Questa non è certamente un'affermazione positiva. Page duBois dice che "women in the world of Odyssey are trapped in cyclical, mythic time ... they belong to an age which Odysseus leaves behind as he makes himself discover 
himself through his journey" (101). La posizione di Pavese, alla luce di quanto riportato dalla duBois nei riguardi di Saffo, è ambigua: per lui essa diventa una figura mitica, un punto fermo dove, misurando il suo grado di potenza maschile, scopre se stesso, la sua condizione di artista e di uomo, che non è poi cosí dissimile da quello della poetessa, al punto da portare avanti il suo discorso nella figura di Rosetta.

Rosetta, come Saffo di Lesbo, vive la sua condizione di donna in una società che va evolvendosi, che comincia a prendere in considerazione le donne come soggetti agenti e non come oggetti. Purtroppo questa forma di libertà all'inizio miete vittime. Forse, un atteggiamento distaccato può renderle indipendenti, esempio Clelia. $\mathrm{Ma}$ anche con un simile atteggiamento, secondo Pavese, che cosa si è conquistato se non la morte, che è un nulla alluccinante? Infatti, la Saffo di "Schiuma d'onda" perde "la serenità olimpica dell'eroe omerico" e "rinuncia al senso positivo dell'esistenza," come dice Della Corte.

La dea del mare è una presenza continua eterna e le onde del mare respirano la sua sostanza. Saffo fa parte di questo movimento ondoso. Se l'aspetto esistenziale è piú facile da accettare per Pavese in quanto scrittore a noi contemporaneo, per gli altri due scrittori, rappresenta una condizione nuova dell'artista nella prima metà dell'Ottocento. Il vuoto esistenziale di Saffo riflette la condizione di un artista che parla una voce nuova. In Grillparzer prende forma di un difetto psicologico perché in fondo tutti l'ammirano considerandola un essere superiore; in Leopardi è brutta e infelice. In Grillparzer se il suicidio rappresenta un riscatto da parte della poetessa da una situazione che l'aveva resa donna, per Leopardi, e anche per Pavese, esso non rappresenta una soluzione in quanto è parte del nulla. Inoltre tutti e tre gli scrittori attaccano alle radici la concezione della serenità degli eroi del mondo ellenico per mettere in evidenza, attraverso la figura di Saffo, la componente dionisiaca che si scontra con quella apolloniana, anche se quest'ultima è mantenuta tale da uno stile contenuto.

Nelle sue poesie, Saffo di Lesbo parla di Afrodite modulando il tema della lontananza, della distanza e del distacco, una dea sorda ai dolori della donna; in Pavese, come del resto gli altri dei, ha un sorriso sereno, distaccato dai dolori degli uomini. In Grillparzer si 
ripropone nella poetessa che torna pura al mare, ma non senza aver preso una coscienza diversa da quello che era la concezione dell'antichità classica nella tradizione greco-tedesca. Nella morte la Saffo di Pavese continua a vivere come onda tormentata. Il vuoto indefinito, "il nulla eterno" del poeta recanese, si ripercuote nel movimento monotono e incessante delle onde pavesiane.

\section{Southern Methodist University}

\section{OPERE CONSULTATE}

DELLA CORTE, Francesco. Saffo, Storia, e Leggenda. Torino: Editore Gheroni, 1950.

DUBOIS, page. "Sappho and Helen." Women in the Ancient World. Ed. John Peradotto \& J.P. Sullivan. Albany: State University of New York Press, 1984. GRILLPARZER, Franz. Sappho. Ed. Walter Rippman. London: The Macmillan Company, 1901.

JENKYNS, Richard. Three Classical Poets. Cambridge, Mass.: Harvard U.P., 1982.

LEOPARDI, Giacomo. "Ultimo canto di Saffo." Canti. Introduzione di G. De Robertis. Firenze: LeMonnier, 1934.

LONARDI, Gilberto. Leopardismo. Firenze: Sansoni, 1974.

PAVESE, Cesare. "Schiuma d'onda," "La rupe." Dialoghi con Leucò. Torino: Einaudi, 1981. . Tra donne sole. Milano: Mondadori, 1981. . Lettere 1945-50. Vol. 2. Torino: Einaudi, 1966.

PIPPIN BRUNETT, Anne. Three Archaic Poets. Cambridge, Mass.: Harvard U.P., 1983.

STEHLE, Eva. "Sappho's Private World." Reflections of Women in Anliquity. Ed. Helene P. Foley. New York: Gordon \& Breach Science Publishers, 1981. 